\title{
A Model for Estimating the Number of Stars for which Terrestrial Planets can be Detected Using Transits
}

\author{
David Koch
}

NASA Ames Research Center, MS245-6, Moffett Field, CA 94035, USA

\begin{abstract}
Borucki \& Summers (1984) have suggested that extrasolar terrestrial planets can be detected by looking for transits. A discussion is presented of a model used to estimate the number and distance of stars for which planets can be detected as a function of spectral type and luminosity class, apparent brightness, planetary size and orbit, and realistic noise sources that must be considered. A choice for an optimum location of the field to search is described given the realistic constraints of a space mission and maximizing the number of available stars.
\end{abstract}

\section{Planetary Transit Detectability Overview}

A study was conducted that showed the most efficient method for monitoring a magnitude limited large sample of stars for transits from space was to use a one-meter aperture telescope with about a $100^{\circ}$ sq. field-of-view (FOV) pointed continuously at a single field. Alternatives considered were to repeatedly repoint a similar telescope at multiple fields with a resultant reduced exposure time per field; use of a larger aperture smaller FOV; and a multiple smaller-aperture "fly's-eye" system monitoring nearly the entire sky and only the bright-est stars. Presented here is a structured approach to the factors that determine the detectability of small brightness changes $(\Delta L / L)$ for a $1-\mathrm{m}, 100^{\circ}$ sq. FOV concept. The details of the process are illustrated in Fig. 1. The transit signal is determined by: 1) the planet size $\left(A_{P}\right)$, and 2) the stellar size $\left(A^{*}\right)$. The noise depends on the: 1) stellar variability $(\nu), 2)$ stellar shot noise $(s), 3)$ background noise $(b)$ and 4 ) photometer noise $(p)$. Differential ensemble photometry is used in the data processing to remove common-mode noise. The low-frequency noise outside the time domain of transits is also filtered out. The orbital semi-major axis $(a)$ and observing interval define the number of transits $\left(N_{T}\right)$. Requiring a detection threshold of $8 \sigma$ from four transits ( $4 \sigma$ for an individual transit) ensures a negligible number of false detections. The minimum detectable planet size as a function of stellar size and planetary orbit can be determined from the equation for the SNR for a given photometer design and assumed stellar variability.

$$
S N R=\frac{(\Delta L / L) \sqrt{N_{T}}}{\sqrt{\nu^{2}+s^{2}+b^{2}+p^{2}}} \cong \frac{\left(A_{p} / A^{*}\right) \sqrt{N_{T} \tau / \tau_{0}}}{\sqrt{\nu_{0}^{2}+1 / n_{0}+b_{0}^{2}+p_{0}^{2}}}=\frac{A_{p} / A^{*}}{\sqrt{a / a_{0}} \sqrt{\nu_{0}^{2}+1 / n_{0}+b_{0}^{2}+p_{0}^{2}}}
$$

Noise terms in Eq. 1 are fractional values. The terms with a zero subscript refer to the noise for a given transit duration $\tau_{0}$ for an orbit of radius $a_{0}$ where $\tau=13 \sqrt{a}$ hours ( $a$ is in AU; Koch \& Borucki 1996). The sign $\cong$ is needed, since not all noise terms vary exactly as the square root of $\tau$. The number of 
photoelectrons counted during the transit is $n_{0}$. For the Kepler Mission (Borucki et al. 2002), the planet detectability for an $\mathrm{m}_{V}=12$ star is shown in Fig. 2.

\section{Model the Number of Stars in the Selected Field of View}

Transits are only detectable if their orbital plane is aligned along the line of sight to the star within an angle equal to the diameter of the star, $2 r^{*}$, divided by the radius of the orbit, $a$. This is true for all orbital plane rotations about the line of sight. These orbital pole positions define a band on the celestial sphere of area, $2 \pi \times 2 r^{*} / a=4 \pi r^{*} / a$ ster. Dividing by the area of a sphere ( $4 \pi$ ster) gives the probability for alignment, $P=0.467 \% r^{*} / a$ (where $r^{*}$ is in solar radii and $a$ in AU) (Boruki \& Summers 1984; Koch \& Boruki 1996). Thus, one needs to monitor tens to hundreds of thousands of stars to expect finding many planets. The star field must also be out of the ecliptic plane to permit continuous year-round observing. One can use the USNO A2.0 catalog to determine the number of stars per sq deg as a function of magnitude for any field. This was done to identify an optimum region centered on RA $19^{h} 41^{m}$, Dec $34^{\circ} 55^{\prime}$. Most star catalogs (SAO, PPM, Hipparcos Input Catalog, etc.) cutoff somewhere around $\mathrm{m}_{V}=9-10$ and only provide spectral type information. Therefore, one needs to model the Galaxy in order to estimate the number of stars as a function of spectral type, luminosity class, and apparent magnitude to $\mathrm{m}_{V}=14$. By fitting the actual number of stars per apparent magnitude for the field to the luminosty function, $\phi(M)$, from Allen (1976), (which provides the number of stars $/ \mathrm{pc}^{3}$ as a function of absolute magnitude and spectral type) one can obtain an estimate of the number of dwarf stars per apparent magnitude and spectral type. Extinction of $A_{v}=0.9 / \mathrm{kpc}$ was included.

\section{Resultant Estimate of Number and Distance of Stars}

By combining the galactic distribution of spectral type and luminosity of stars with the detectability analysis as a function of planetary size and orbit; stellar size and brightness; and 1-m aperture photometer performance, a solar-like variability of $10 \mathrm{ppm}$ and space mission duration of $4 \mathrm{yr}$; one can calculate the estimated number of stars for which various size planets can be detected (Fig. 3a). Mass in Fig. 3b is based on using a terrestrial density of 5.5. Of particular interest are the terrestrial planets in 1-yr orbits about solar-like stars, since these are considered to be planets in the HZ. Most of the parent stars for $M=1 M_{\oplus}$ are $\leq 400 \mathrm{pc}$. Shorter period orbits allow the use of more stars for detection of a given planet size or the ability to detect even smaller planets for the given stellar sizes as can be seen from Eq. 1. The number of planets will be measured by the Kepler Mission (Borucki et al. 2002) and the frequency distributions found by taking into account the dependencies presented here.

\section{References}

Allen, C. W. 1976, Astrophysical Quantities $3^{\text {rd }}$ ed., (Athlone Press), 247

Borucki, W. J., \& Summers, A. L. 1984, Icarus 58, 121

Borucki, W. J., et al. 2002, SPIE Conf 4854

Koch, D., \& Borucki, W. 1996, in Circumstellar Habitable Zones, ed. L. Doyle, (Menlo Park: Travis House Pub), 229 


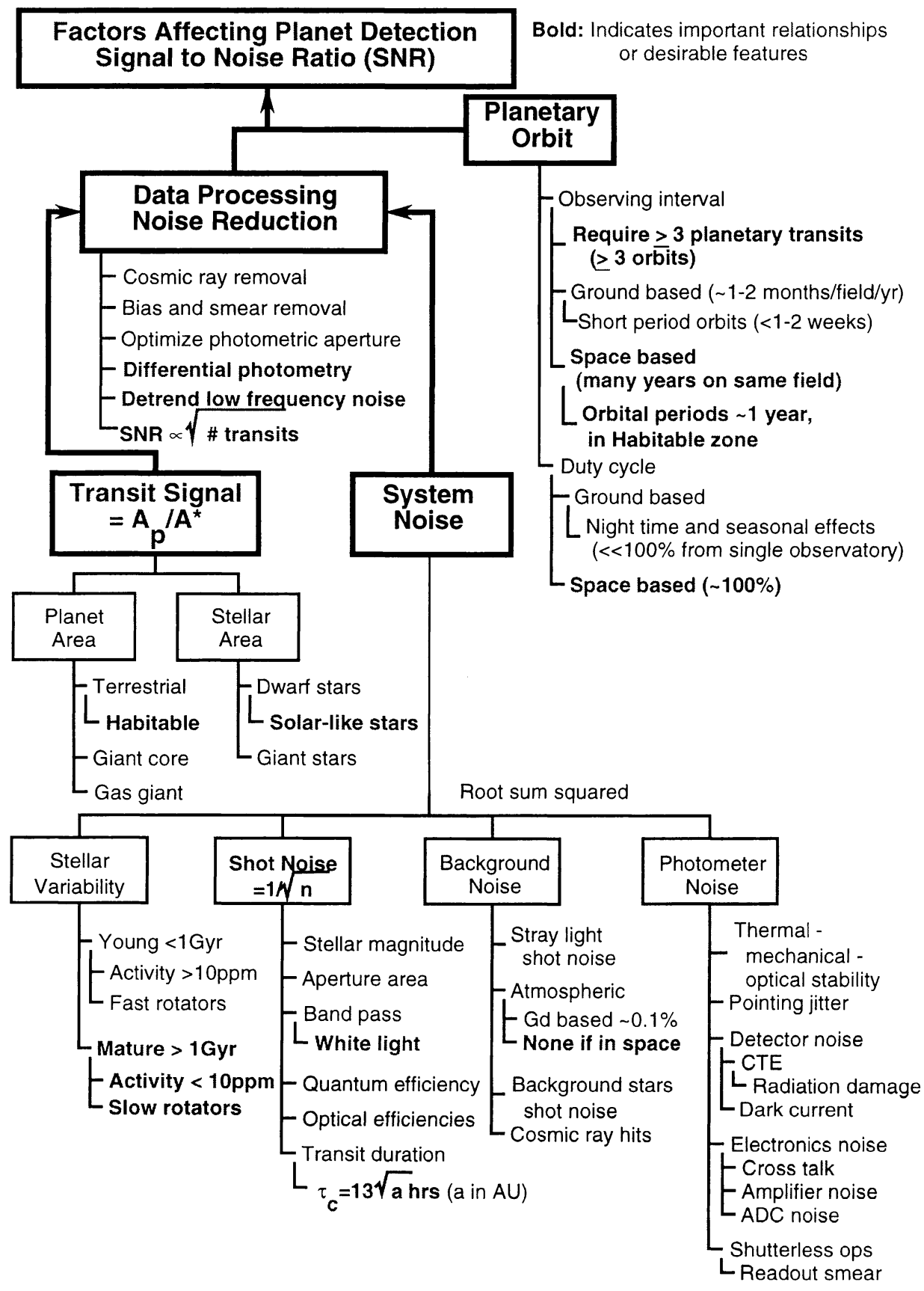

Figure 1. The most significant factors affecting the detection of planets using the transit method. Ground based observing is constrained to detection of giant planets only. Space based transit detection can find planets substantially smaller than the Earth in the HZ. 


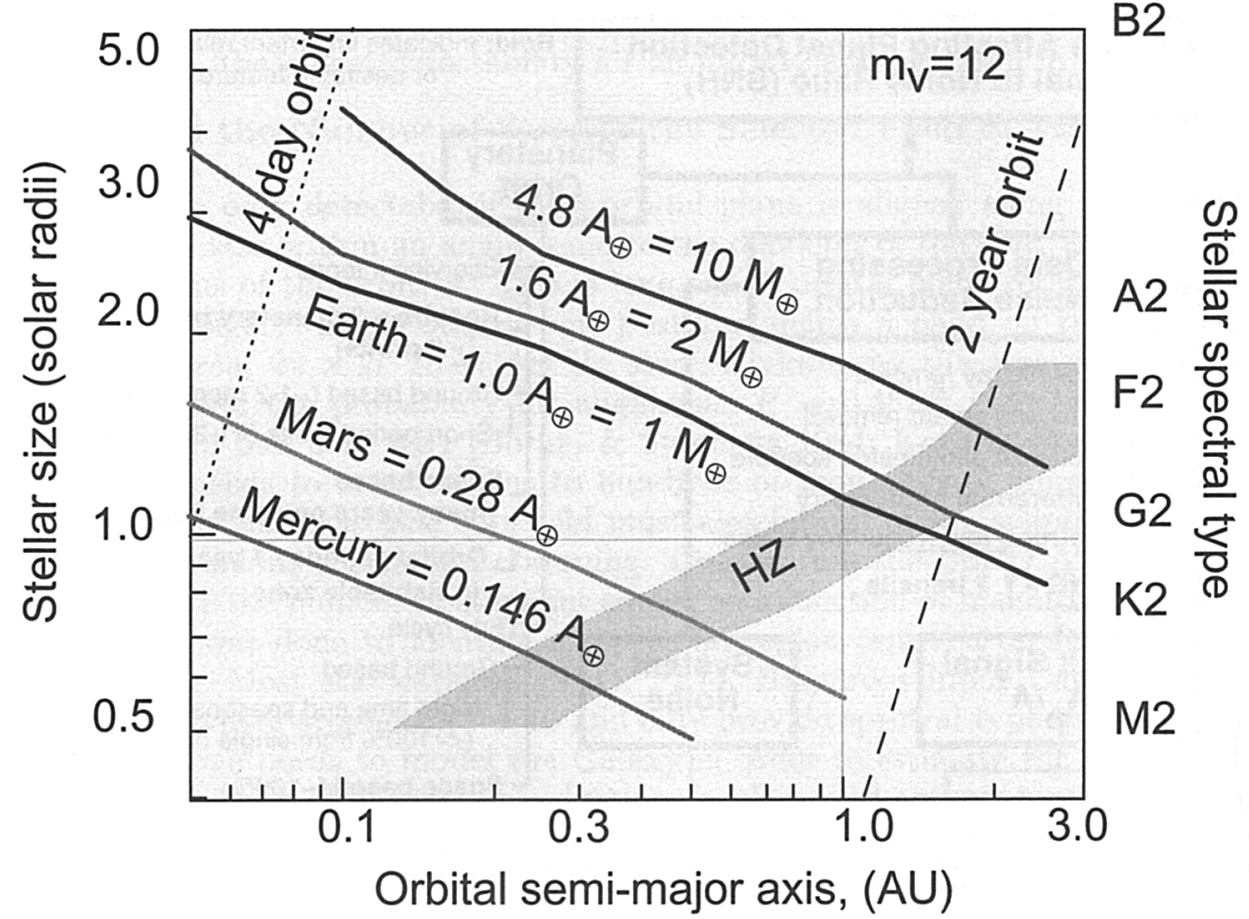

Figure 2. The detectable planet size is shown for a nearly central transit. An 0.8 Earth area planet can be detected for a solar-like star $(\mathrm{G} 2 \mathrm{~V})$ at $1 \mathrm{AU}$. Detectability is based on a total SNR $=8 \sigma$ and $=3 \sigma$ transits in 4 yrs.
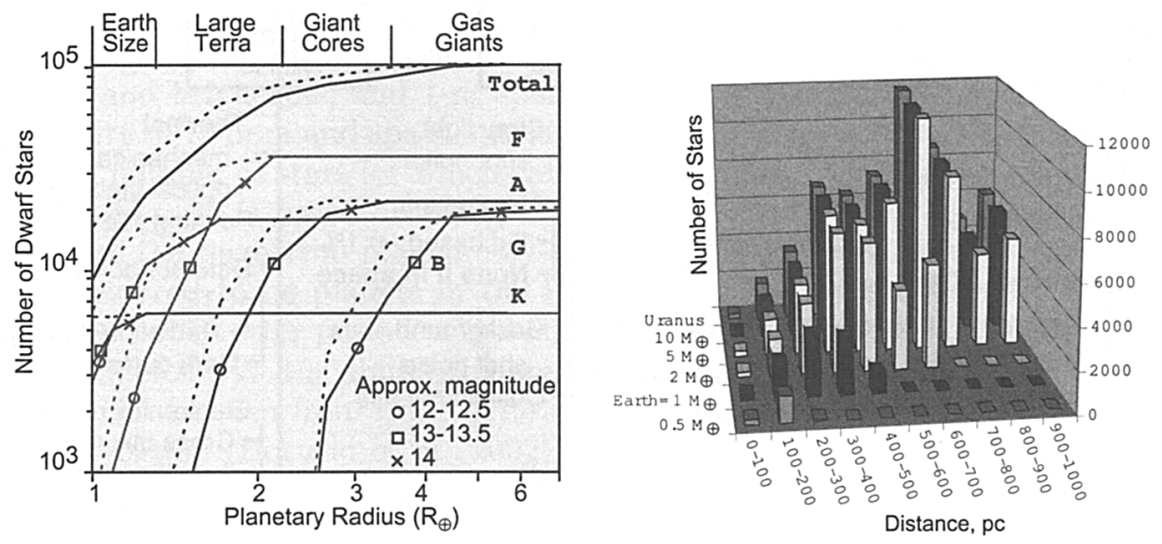

Figure 3. (a) Number and spectral type of dwarf stars versus detectable planet size. Solid lines are for $\mathrm{SNR} \geq 8 \sigma$ near grazing transits in 1-yr orbit. Dashed lines are for a nearly central transit in 1-year orbit. (b) Number and distance of dwarf stars for various planet masses. Based on 1-yr orbit, SNR $\geq 8 \sigma, 4$ transits lasting $80 \%$ of a central transit, and monitoring of 100000 stars for 4 yrs. 